\title{
Hydrodynamic characteristics for flow around wavy wings with different wave lengths
}

\author{
Mi Jeong Kim ${ }^{1}$, Hyun Sik Yoon ${ }^{2}$, Jae Hwan Jung ${ }^{1}$, Ho Hwan Chun ${ }^{2}$ and Dong Woo Park ${ }^{3}$ \\ ${ }^{1}$ Department of Naval Architecture and Ocean Engineering, Pusan National University, Busan, Korea \\ ${ }^{2}$ Global Core Research Center for Ships and Offshore Plants, Pusan National University, Busan, Korea \\ ${ }^{3}$ Hyundai Heavy Industries, Ulsan, Korea
}

\begin{abstract}
The present study numerically investigates the effect of the wavy leading edge on hydrodynamic characteristics for the flow of rectangular wings with the low aspect ratio of 1.5. Five different wave lengths at fixed wavy amplitude have been considered. Numerical simulations are performed at a wide range of the angle of attack $\left(0^{\circ} \leq \alpha \leq 40^{\circ}\right)$ at one Reynolds number of $10^{6}$. The wavy wings considered in this study did not experience enough lift drop to be defined as the stall, comparing with the smooth wing. However, in the pre-stall region, the wavy wings reveal the considerable loss of the lift, compared to the smooth wing. In the post-stall, the lift coefficients of the smooth wing and the wavy wings are not much different. The pressure coefficient, limiting streamlines and the iso-surface of the spanwise vorticity are also highlighted to examine the effect of the wave length on the flow structures.
\end{abstract}

KEY WORDS: Wavy leading edge; Wave length; Low aspect ratio wing; Lift; Stall.

\section{INTRODUCTION}

The modification of the leading edge that mimics the humpback whale flipper, which is believed to significantly help the whale to execute rolls and loops under water, have been considered to increase the lift of the wing and improve the wing stall characteristics. Fish and Battle (1995) reported that the humpback whale flipper has a wing-like, high aspect ratio planform with rounded tubercles located along the flipper's leading edge. The morphology and arrangement of the tubercles suggest that they act as improved lift devices to control flow around the flipper and to maintain lift at a high angle of attack. Inspired by this uncommon leading edge geometry of the humpback whale flipper, Watts and Fish (2001) numerically investigated the effect of the leading edge tubercles on the forces acting on the wings at only one angle of attack of $10^{\circ}$ by using a panel method. They found an augmentation in lift and a reduction of drag in comparison with a smooth wing, resulting in confirming the suggestion of Fish and Battle (1995).

In contrast to Watts and Fish (2001) who employed a three-dimensional panel method code to investigate a rectangular wing with and without tubercles, Miklosovic et al. (2004) experimentally examined the load characteristics of a threedimensional idealized humpback flipper in a wind tunnel. The tested models are similar in planform geometry to the humpback whale flipper. One model had tubercles along the leading edge, while the other had no tubercles and was used as a baseline for comparison. They found that a $6 \%$ increase in the maximum lift and a $40 \%$ increase in the stall angle were achieved for the model with the tubercles. They also showed that the presence of the tubercles decreases the overall drag in the range of the

Corresponding author: Hyun-Sik Yoon

e-mail:lesmodel@pusan.ac.kr 
angle of attack of $11^{\circ} \leq \alpha \leq 18^{\circ}$. This work is extended in Murray et al. (2005) to include sweep angles of $15^{\circ}$ and $30^{\circ}$ with similar results. Furthermore, Miklosovic et al. (2007) considered the a full-span rectangular wing and experimented the effect of a leading edge tubercle on the load characteristics of a full-span rectangular wing and a semi-span wing, which is based on the idealized humpback whale flipper geometry. They found that the leading edge scallop reduces lift and increases the drag of the full-span rectangular wing, while the semi-span wing produces an opposite trend. This suggests that tubercle effects may be coupled with planform shape and Reynolds number effects.

Recently, the systematical analysis by considering the tubercle particulars has been carried out by several researches (Custodio, 2007; Johari et al., 2007). Custodio (2007) and Johari et al. (2007) experimentally examined the effects of wavelength and wave amplitude of tubercles along the leading edge on the performance of NACA $63_{4}-021$ hydrofoils. The performance of the tubercles hydrofoils, with amplitude ranging from $0.025 \mathrm{C}$ to $0.12 \mathrm{C}$ and wavelength ranging from $0.25 \mathrm{C}$ to $0.5 \mathrm{C}$, are investigated by comparing them with the smooth hydrofoil. They reported that the stall of the tubercle hydrofoils is less abrupt than that of the smooth hydrofoil. In the pre-stall region of the smooth hydrofoil, tubercles reduce the lift. However, in the post-stall region, the tubercles revealed about a 50\% higher lift than the baseline hydrofoil. They also suggested that the wavelength and the wave amplitude play a minor role and a significant role, respectively, in the load characteristics of the foils.

The previous studies mainly concentrated on the idealized flipper or full-span rectangular wing and mainly present not the flow structures such as pressure distribution and vortical structures around the wavy wing but the exerted forces on the wing. Also, it is difficult to find previous studies that have dealt with the semi-span rectangular wing with low aspect ratio, which is the ratio of the span of the wing to its mean chord length.

Recently, author and coworkers (Yoon et al., 2011) investigated numerically the effect of the wavy leading edge on hydrodynamic characteristics for three-dimensional viscous flow around a wing. Yoon et al. (2011) considered the semi-span rectangular wing with the low aspect ratio of 1.5 which falls into the ratio range of the rudder of the ship. They focused on the effect of the waviness ratio on the hydrodynamic characteristics for flow around the wing over a wide range of angles of attack. In addition, they provided the mean flow structures such as pressure distribution, limiting streamlines and vortical structures around the wings with and without the waviness according to the angle of attack, even though their results obtained under the assumption of the steady flow. Yoon et al. (2011) showed that most of waviness ratio present earlier occurrence of the stall than the smooth wing, except the lowest waviness ratio considered in their study. In the post-stall region based on the smooth wing, lift of wings with waviness ratios except the lowest waviness ratio recovered and became larger than those of the smooth wing and the lowest waviness ratio.

This study aims at investigating the effect of the wave length on the hydrodynamic characteristics for flow around the wing over a wide range of angles of attack by considering five different waviness ratios. The present study also considers the semispan rectangular wing with the low aspect ratio of 1.5 like Yoon et al. (2011). Eventually, the computational results of body forces, pressure coefficient, limiting streamlines and vortical structures around a wavy wing with different wave lengths are compared with those around a smooth wing in a wide range of angle of attack.

Even though the present results obtained under the same assumption of the steady flow as Yoon et al. (2011), the relative comparison of the exerted forces on the smooth wing and the wavy wings with different wave lengths according to the angle of attack is expected to provide valuable information. In addition, this study will contribute to supplement the previous work (Yoon et al., 2011).

\section{COMPUTATIONAL DETAILS}

The commercial CFD package is employed for all numerical predictions. The present study considers the 3D problem with the assumption of the steady incompressible viscous flow in order to simplify the physical problem. Therefore, the governing equations describing the steady incompressible viscous flow field in the present study are the continuity and Reynolds averaged Navier-Stokes equations:

$$
\frac{\partial U_{i}}{\partial x_{i}}=0
$$




$$
\frac{\partial}{\partial x_{j}}\left(\rho U_{i} U_{j}\right)=-\frac{\partial P}{\partial x_{i}}+\frac{\partial}{\partial x_{j}}\left[\mu\left(\frac{\partial U_{i}}{\partial x_{j}}+\frac{\partial U_{j}}{\partial x_{i}}\right)\right]+\frac{\partial}{\partial x_{j}}\left(-\rho \overline{u_{i}^{\prime} u_{j}^{\prime}}\right)
$$

where $x_{i}$ are Cartesian coordinates, $U_{i}$ are the corresponding velocity components, $\rho$ is the density, $\mu$ is the viscosity, and $P$ is the pressure. Also, $-\rho \overline{u_{i}^{\prime} u_{j}^{\prime}}$ in equation (2) is the Reynolds stress term which has been closed by using the realizable $k-\omega$ model. The realizable $k-\omega$ model is employed as the turbulence mode. It is improved predictive capabilities compared to the standard $k-\omega$ model. Also, realizable $k-\omega$ model has the ability to resolve more accurately and more reliably for a wider class of flow such as the adverse pressure gradient flows and flow around the airfoils.

This model ensures that both the near-wall and far-field zones are appropriately solved because an additional cross-diffusion term in the $\omega$ equation was included. The equations are solved by the SIMPLE algorithm with a QUICK spatial discretization scheme based on a finite volume method. The convergence criterion of $10^{-6}$ has been used for the present simulations. Further details of the implementation can be found in the FLUENT (2005) manuals. The present numerical methods have successfully used in authors' previous study (Yoon et al., 2011) to see the effect of the waviness ratio on the hydrodynamic characteristics for flow around the wing over a wide range of angles of attack.

Fig. 1 shows the coordinate system, the computational domain and boundary conditions of the problem. A fixed Cartesian coordinate system $(x, y, z)$ is used with the origin located at the end of the wing tip. The $x$-axis is aligned with the inlet flow direction. The $z$-axis is parallel to the wing spanwise direction and the $y$-axis is perpendicular to both the $x$ and $z$ axes. The size of the computational domain formed as a cylindrical shape is defined by the radius of $10 C$ and the height of $4.5 C$, where $C$ represents the mean chord length of the wing.

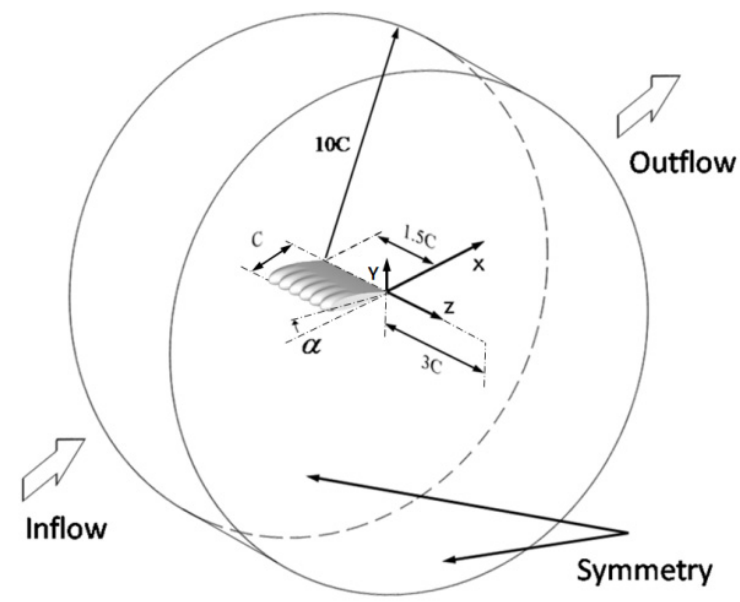

Fig. 1 Computational domain and coordinate system along with boundary conditions.

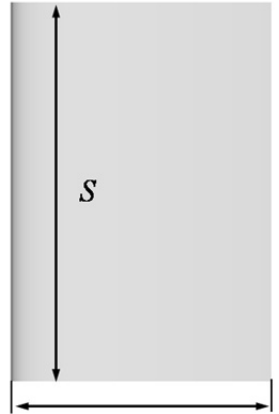

C

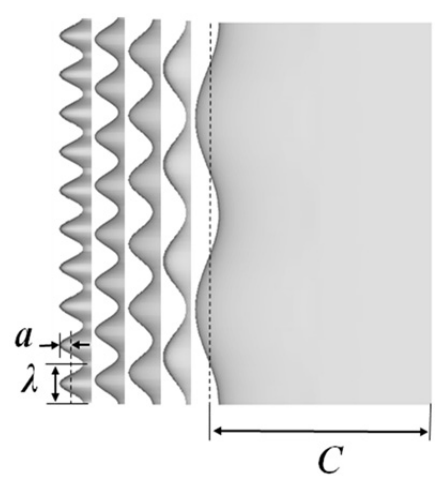

(b)

Fig. 2 Top view of geometries of (a) the rectangular wing and (b) wavy wings with different wave lengths. 


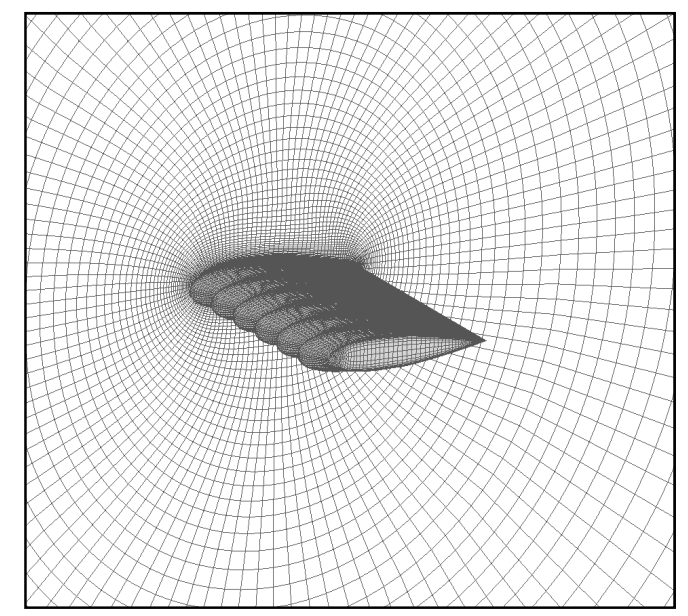

Fig. 3 Grid system near the wavy wing of $\lambda=S / 6$.

A no-slip boundary condition is imposed on the wing surface while the symmetry condition is set for the far-field boundary. The uniform flow with the free stream velocity $\left(U_{\infty}\right)$ and low turbulent intensity are imposed on the inflow boundary condition. The convective condition is applied on the outflow boundary. The computations for the flow around the NACA 0020 wing section with the span $(S)$ of $1.5 C$ are performed at the Reynolds number $\left(\operatorname{Re}=\rho U_{\infty} C / \mu\right)$ of $10^{6}$ for the wide range of angle of attack of $0^{\circ} \leq \alpha \leq 40^{\circ}$.

Fig. 2 shows schematic diagrams of the rectangular wing and the wavy wing. The wavy leading edge has a sinusoidal variation in the cross sectional area along the spanwise direction, which is described by:

$$
C(z)=C+a \cos (2 \pi z / \lambda)
$$

where $C(z)$ denotes the local chord length of the profile, $a$ is the wavy amplitude and $\lambda$ is wave length. In the present study, the wavy amplitude have fixed values of $0.05 C$. The hydrodynamic characteristics of the flow around the wavy wing are examined at five different wave lengths of $S / 2, S / 4, S / 6, S / 8$ and $S / 10$.

Fig. 3 shows the grid system near the wavy wing of $\lambda=S / 6$. The averaged minimum grid spacing on the wing surface of $2 \times 10^{-5}$ corresponds to $y^{+} \approx 1$ as the wall unit. The grid is continuously coarsened when the distance from the wing is increased. The total grid number used in this study is 2.3 million. The present grid number is comparable to the grid number of Yoon et al. (2011) who also investigated numerically the effect of the wavy leading edge on hydrodynamic characteristics for three-dimensional viscous flow under the assumption of the steady flow.

Regarding to the verification of the present numerical methodologies, as above mentioned, the present numerical methods and the grid system follow the authors' previous study (Yoon et al., 2011) for the problem of the wavy leading edge. Thus, the validation of the present numerical method can be refereed from Yoon et al. (2011) who followed Molland and Turnock's (1993) experimental conditions of the smooth rectangular wing with the NACA 0020 section and $A R=1.5$, with the angle of attack ranging from $0^{\circ}$ to $40^{\circ}$ and $\mathrm{Re}=10^{6}$. The lift and drag coefficients $\left(C_{L}, C_{D}\right)$ of Yoon et al. (2011) agreed well with those of Molland and Turnock's (1993) experiment.

\section{RESULTS AND DISCUSSION}

\section{Lift and drag coefficients}

The pressure and viscous components of the lift coefficient $\left(C_{L}\right)$ and drag coefficient $\left(C_{D}\right)$ of the smooth wing as a function of the angle of attack were presented by Yoon et al. (2011). They showed that the pressure component is predominant in both the lift coefficient and drag coefficient. Thus, they focused on the pressure effect on the force coefficients. Consistently, this study also concerns mainly the pressure effect on the force coefficients according to the wave length. 
The variation of $C_{L}$ according to the angle of attack for the wavy wings with different wave length and the smooth attack in the range of $0^{\circ} \leq \alpha \leq 8^{\circ}$, regardless of the wave length, the effect of the wavy leading edge on the lift coefficient is almost negligible. However, when $\alpha>8^{\circ}$, the discrepancy of $C_{L}$ between the smooth wing and the wavy wings is significant.

In the case of the smooth wing, $C_{L}$ increases monotonically with increasing $\alpha$ up to $20^{\circ}$, where $C_{L}$ has a maximum value. As $\alpha$ increases continuously to $20^{\circ}, C_{L}$ decreases dramatically. Further increasing $\alpha$, wing is plotted in Fig. 4(a). In general, for the low angles of $C_{L}$ is almost saturated.

In contrast to the smooth wing, the wavy wings show very small the reduction of $C_{L}$ in the range of $12^{\circ} \leq \alpha \leq 16^{\circ}$, even though $\alpha$ revealing the lift loss depends on the wavy length in this range of $\alpha$. After undergone the slight drop of the lift, with increasing $\alpha$ to $24^{\circ}, C_{L}$ augments again. As $\alpha$ increases continuously from $24^{\circ}$, the variation of $C_{L}$ is almost negligible. Consequently, the wavy wings considered in this study don't present enough drop of $C_{L}$ to be defined as the stall, comparing with the smooth wing as shown in Fig. 4(a).

In the range of $24^{\circ} \leq \alpha<40^{\circ}$, the cases of $\lambda=S / 2, \lambda=S / 4$ and $\lambda=S / 6$ provide slightly larger $C_{L}$ than the smooth wing. This pattern of $C_{L}$ in the post-stall region $\left(24^{\circ} \leq \alpha<40^{\circ}\right)$ based on the smooth wing is consistent with the finds of Johari et al. (2007) and Yoon et al. (2011) which showed that $C_{L}$ for the wavy wings recovered and became larger than those of the smooth wing in this region.

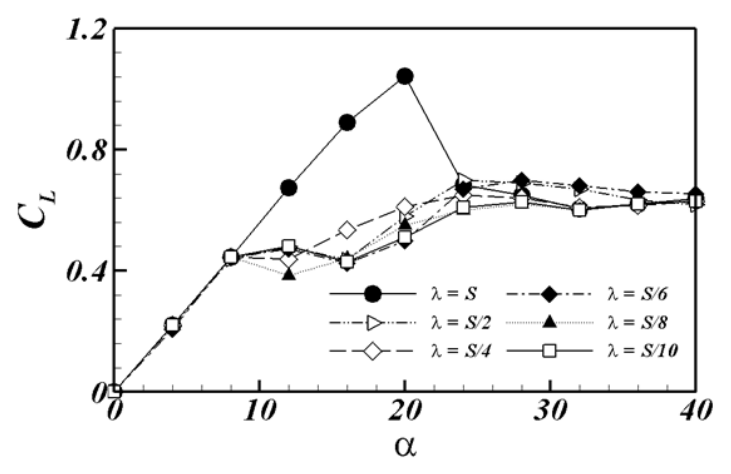

(a)

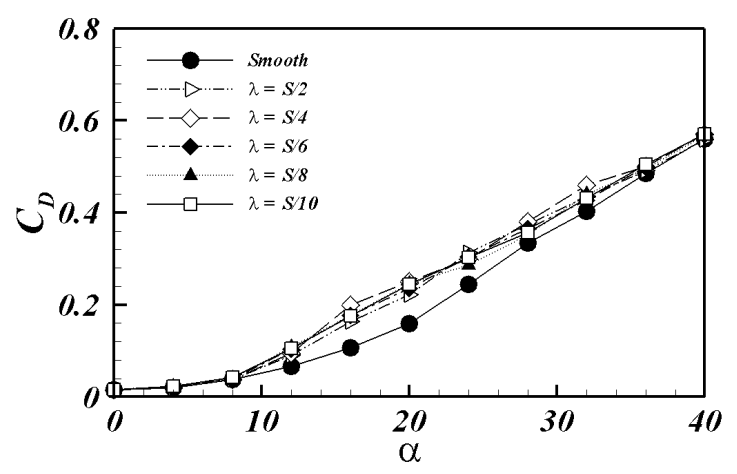

(b)

Fig. 4 (a) Lift coefficient and (b) drag coefficient of the wavy wing and the smooth wing as according to the angle of attack.

Fig. 4(b) shows the drag coefficient $\left(C_{D}\right)$ as a function of $a$ for the smooth wing and wavy wings. In general, for the low angles of attack in the range of $0^{\circ} \leq \alpha \leq 8^{\circ}$, regardless of the wave length, the wavy wings show about the same value of $C_{D}$ with the smooth wing. However, when $12^{\circ} \leq \alpha<24^{\circ}$, all wavy wings start to provide the considerable increase of $C_{D}$ to the smooth wing, especially $\lambda=S / 4$. In the post-stall region $\left(24^{\circ} \leq \alpha<40^{\circ}\right)$, except the case of $\lambda=S / 4$, the wavy wings diminish the increment of $C_{D}$ to the smooth wing.

\section{Distribution of the pressure coefficient}

Here, three different angles of attack of $12^{\circ}, 20^{\circ}$ and $24^{\circ}$ are representatively considered, where the variation of the hydrodynamic forces are apparent according to the wave length. Fig. 5 shows the distribution of $C_{P}$ on the upper surface for the smooth wing and the wavy wings at $\alpha=12^{\circ}$. For the smooth wing, the low values of $C_{P}$ distribute near the leading edge. The lowest value of $C_{P}$ appears near the wing root, and $C_{P}$ then increases towards the trailing edge as shown in Fig. 5(a), since the effective angle of attack becomes smaller from the root to the tip of the wing owing to the downwash flow (van Nierop et al., 2008). Additionally, the lower values of $C_{P}$ occupy a broader area at near the leading edge unlike the wavy wings. Consequently, $C_{L}$ and $C_{D}$ for the smooth wing at $\alpha=12^{\circ}$ have the largest and smallest values as revealed in Figs. 4 (a) and 4(b), respectively. 


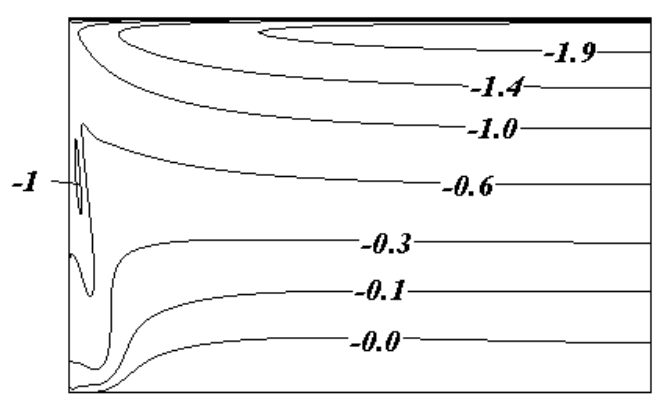

(a)

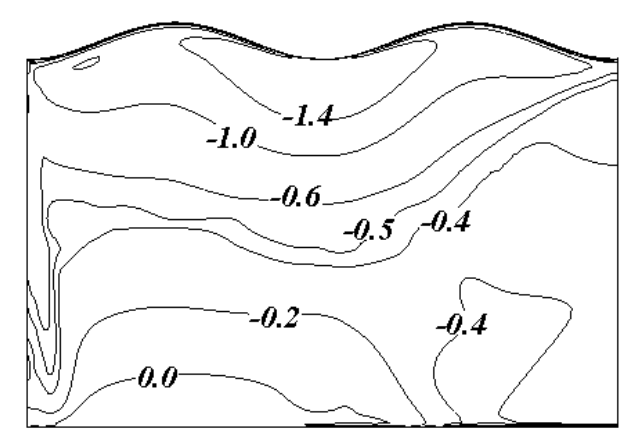

(b)

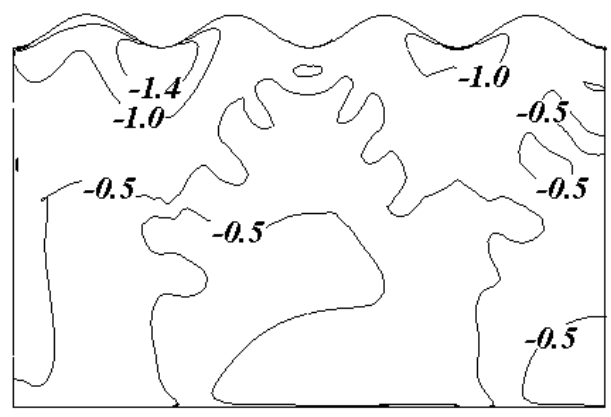

(c)

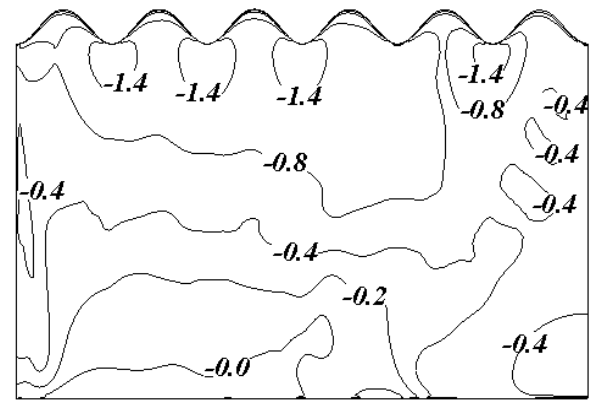

(d)

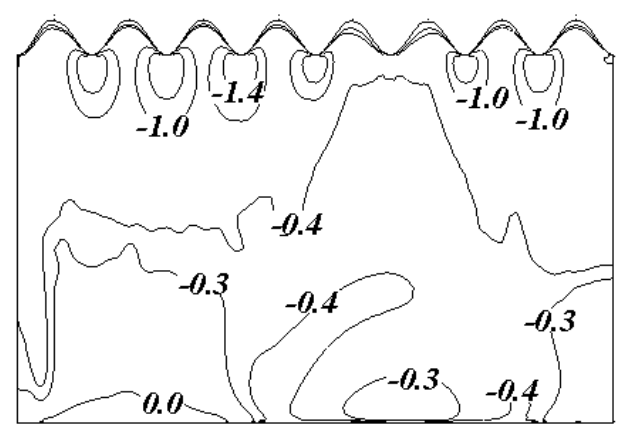

(e)

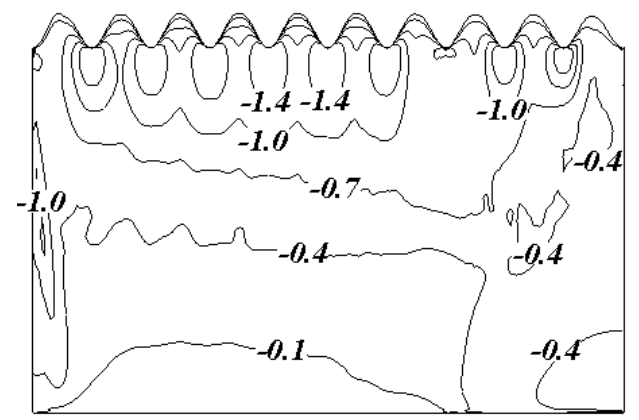

(f)

Fig. 5 Distribution of pressure coefficient $\left(C_{P}\right)$ on the upper surface at $\alpha=12^{\circ}$ for different wings;

(a) smooth wing, (b) $\lambda=S / 2$, (c) $\lambda=S / 4$, (d) $\lambda=S / 6$, (e) $\lambda=S / 8$, and (f) $\lambda=S / 10$.

In the cases of $\lambda=S / 2, S / 4, S / 6, S / 8$ and $S / 10$, the relatively high values of $C_{P}$ diverge from the first trough on the inboard side to the outboard side of the wing tip as shown in Figs. 5(b) (f). Especially, in the cases of $\lambda=S / 4$ and $S / 8$, the relatively high values of $C_{P}$ become close to the leading edge with an almost symmetrical distribution about the third trough and the fourth trough on the inboard side as shown in Figs. 5(c) and 5(e), respectively. Thus, the high pressure distributes more widely in the central region than the other wings. Yoon et al. (2011) also presented the divergence of the pressure distribution from the trough forms relatively high pressure region, leading to the lift loss.

When $\alpha=20^{\circ}$, the smooth wing presents a wider distribution of the lower $C_{P}$ from the root side of the leading edge as shown in Fig. 6(a) owing to the augmentation of the suction effect. On the inboard side, the helical nature of the limiting streamlines of the wall shear components and the vortical structures are formed at this angle of attack. Thus, as $\alpha$ increases to $20^{\circ}$, the lower values of $C_{P}$ occupy a broader area, which contributes to the augmentation of $C_{L}$.

At $\alpha=20^{\circ}$, the wavy wings with different wavy lengths reveal wide distribution of relatively higher $C_{P}$ on the surface than the smooth wing, as shown in Figs. 6(a) (f). In cases of the wavy wings, the divergence of $C_{P}$ distribution from the wave 
trough is preserved and predominant to the formation of $C_{P}$ on the surface as observed from Figs. 6(b) (f), which is appeared at lower $\alpha=12^{\circ}$ in Figs. 5(b) (f).

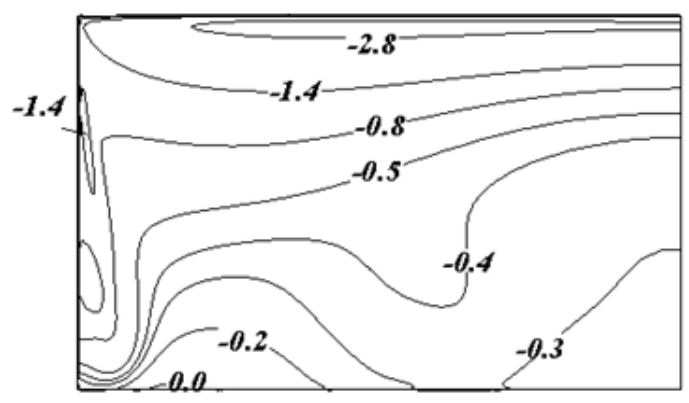

(a)

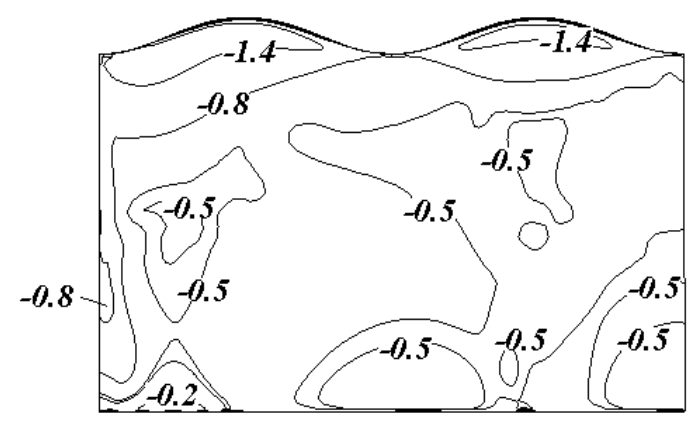

(b)

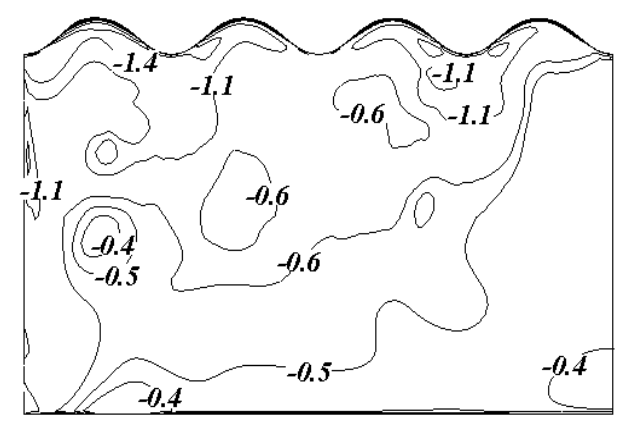

(c)

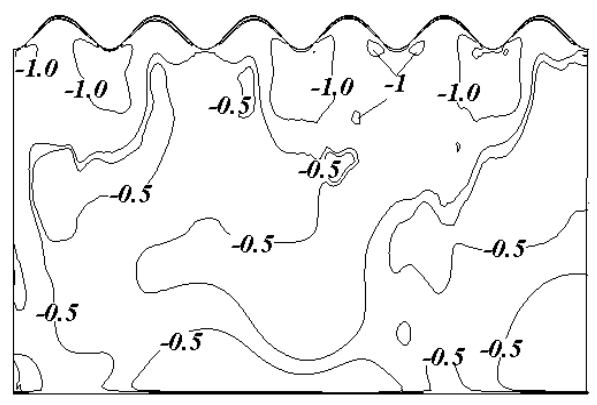

(d)

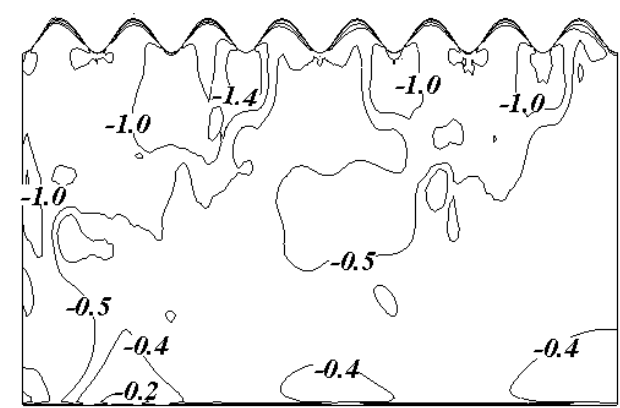

(e)

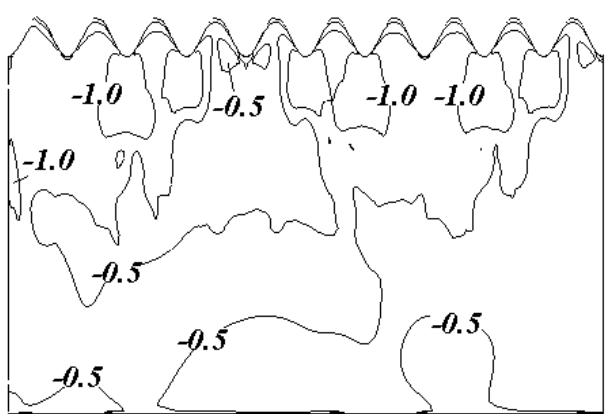

(f)

Fig. 6 Distribution of pressure coefficient $\left(C_{P}\right.$ ) on the upper surface at $\alpha=20^{\circ}$ for different wings; (a) smooth wing, (b) $\lambda=S / 2$, (c) $\lambda=S / 4$, (d) $\lambda=S / 6$, (e) $\lambda=S / 8$, and (f) $\lambda=S / 10$.

Consequently, the values of $C_{L}$ for all wavy wings at $\alpha=20^{\circ}$ are much smaller than the smooth wing in Fig. 4(a). Fig. 7 shows the distribution of $C_{P}$ on the upper surface of the smooth wing and the wavy wings at $\alpha=24^{\circ}$. For the smooth wing, at $\alpha=24^{\circ}$ in the post stall region, $C_{P}$ leads to the adverse pressure gradient, resulting in the leading edge separation. As the wave length decreases continuously, the high values of $C_{P}$ diverge from the first trough on the inboard side to the outboard side of the wing tip as shown in Figs. 7(b) (f). However, in the cases of $\lambda=S / 2$ and $S / 4$, lower pressures occupy a wider area near the central region and the leading edge. Consequently, all the cases are generally similar distribution but the cases of $\lambda=S / 2$ and $S / 4$ are the large among smooth wing and the other wavy wings considered in this study as shown in Fig. 4(a). In addition, the force coefficient of $C_{D}$ is the larger than smooth wing as shown in Fig. 4(b). 


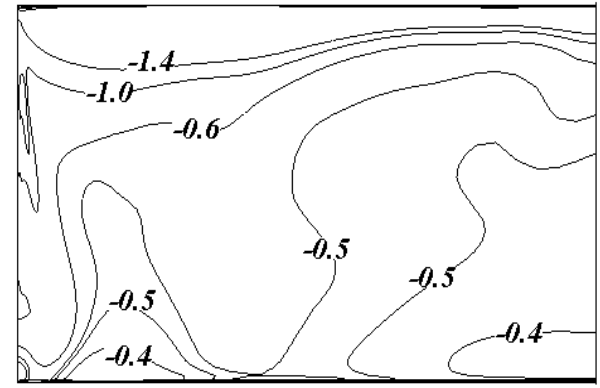

(a)

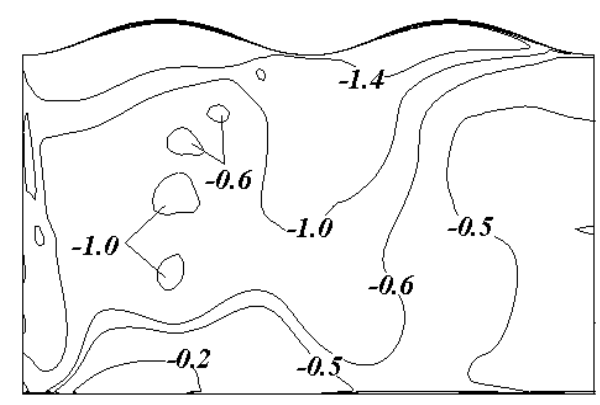

(b)

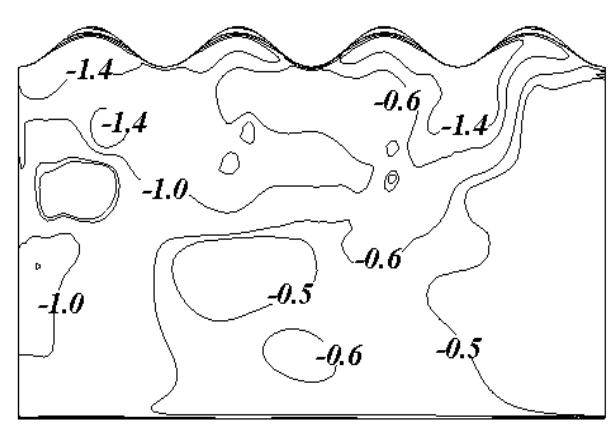

(c)

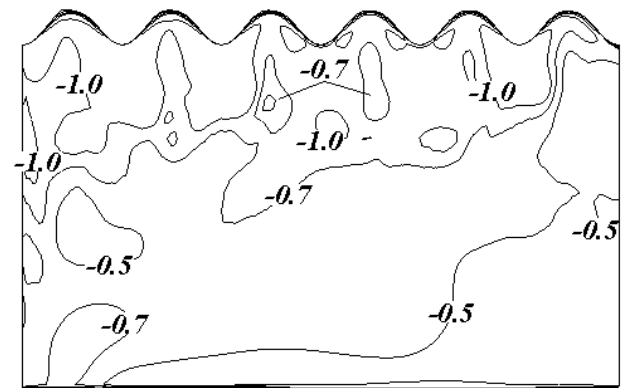

(d)

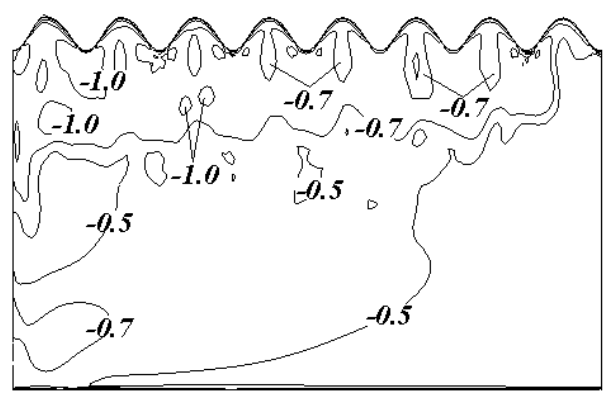

(e)

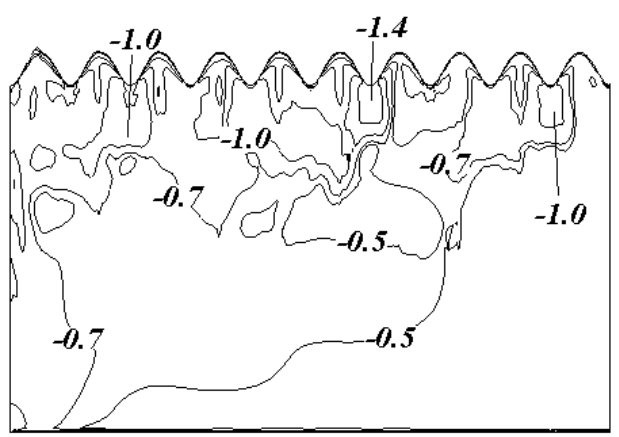

(f)

Fig. 7 Distribution of pressure coefficient $\left(C_{P}\right)$ on the upper surface at $\alpha=24^{\circ}$ for different wings; (a) smooth wing, (b) $\lambda=S / 2$, (c) $\lambda=S / 4$, (d) $\lambda=S / 6$, (e) $\lambda=S / 8$, and (f) $\lambda=S / 10$.

\section{Limiting streamlines and the iso-surface of the spanwise vorticity}

In order to analyze the effect of the flow structures on the hydrodynamic forces observed in Figs. 4(a) and 4(b), the limiting streamlines (the skin friction lines) on the upper surfaces and the iso-surfaces of the spanwise vorticity are plotted in Figs. 8 13 in order to visualize the flow. Fig. 8 and 9 show the limiting streamlines and the iso-surface of the spanwise vorticity on the upper surfaces of the wing, respectively, for the smooth wing and the wavy wings at $\alpha=12^{\circ}$. In the case of smooth wing, excepting tip vortex, the limiting streamlines and iso-surface of the spanwise vorticity show that the flows slightly tilt to the root side of the trailing edge as shown in Figs. 8(a) and 9(a). At this $\alpha=12^{\circ}$, waviness on the leading edge considerably modifies the limiting streamlines on the upper surface. Generally, for wavy wings, the separation lines (or convergence lines) start from the first trough on the inboard side and propagate towards the inboard and outboard. Between the separation ines, the flow returns from the trailing edge to the leading edge as shown in Figs. 8(b) (f). Especially, in the cases of $\lambda=S / 4$ and $S / 8$, the limiting streamlines spread to the transverse direction as shown in Figs. 8(c) and 8(e), respectively. This pattern of the limiting streamline correlates with the relatively high $C_{P}$ distribution in the centre of the wing as previously shown in Fig. 6 . The existence of the return flow within the diverged convergence lines of the limiting streamlines is identified by the large isosurface of the spanwise vorticity as shown in Figs. 9(b) (f) for all wavy wings. 


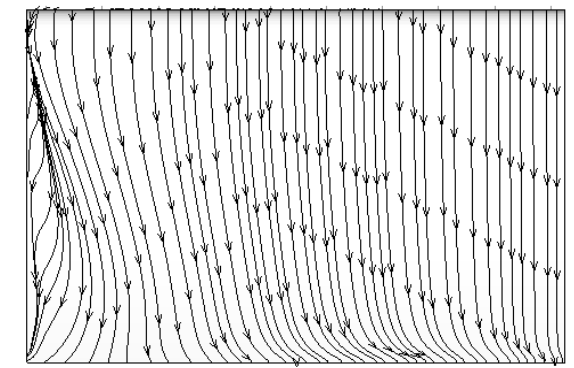

(a)

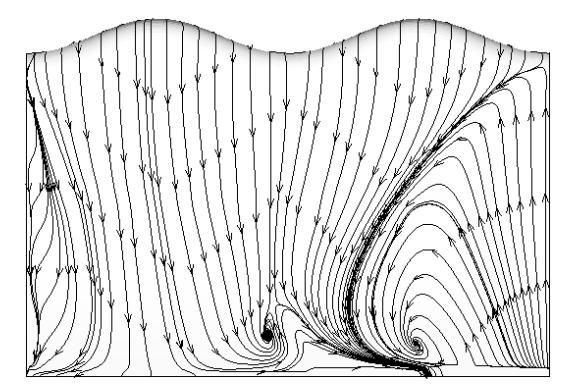

(b)

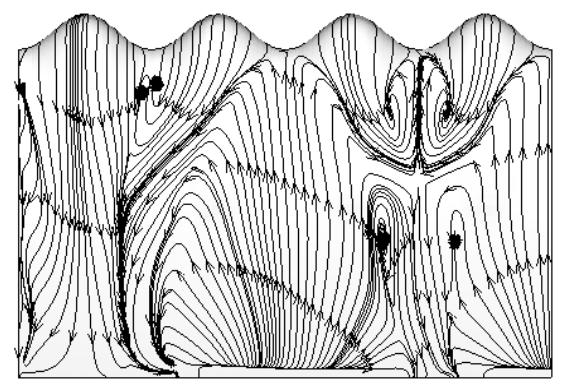

(c)

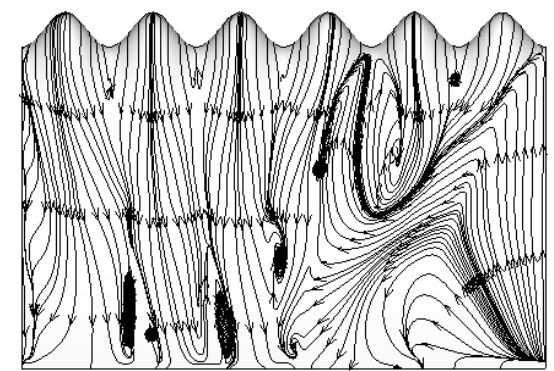

(d)

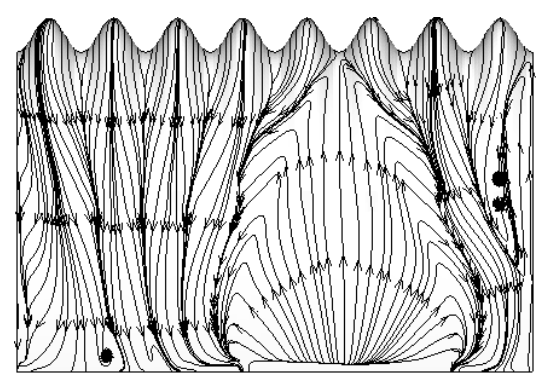

(e)

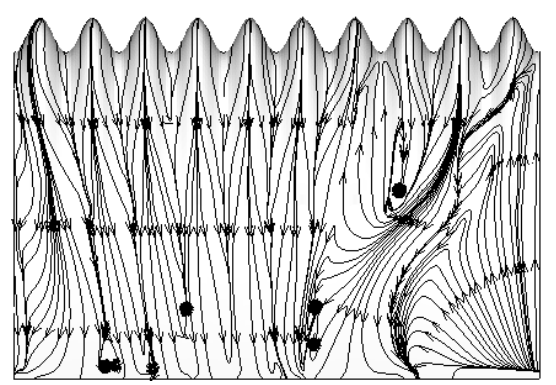

(f)

Fig. 8 Limiting streamlines on the upper surface at $\alpha=12^{\circ}$ for different wings; (a) smooth wing, (b) $\lambda=S / 2$, (c) $\lambda=S / 4$, (d) $\lambda=S / 6$, (e) $\lambda=S / 8$, and (f) $\lambda=S / 10$.

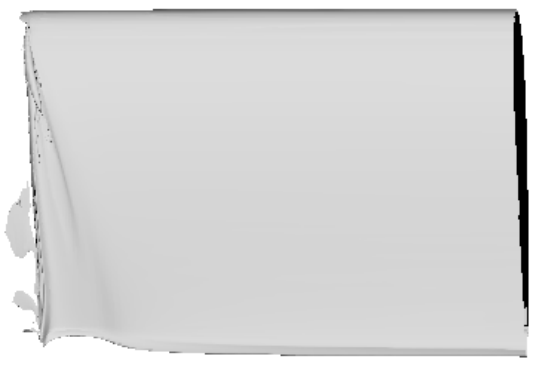

(a)

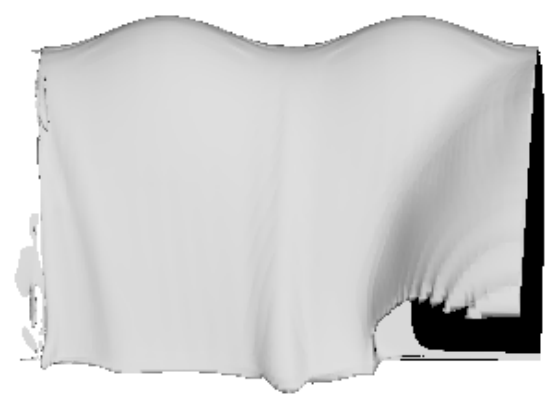

(b)

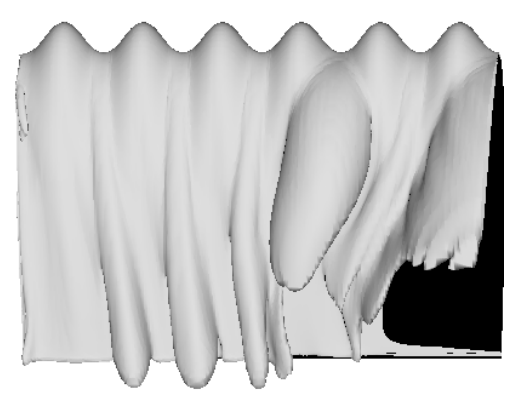

(d)

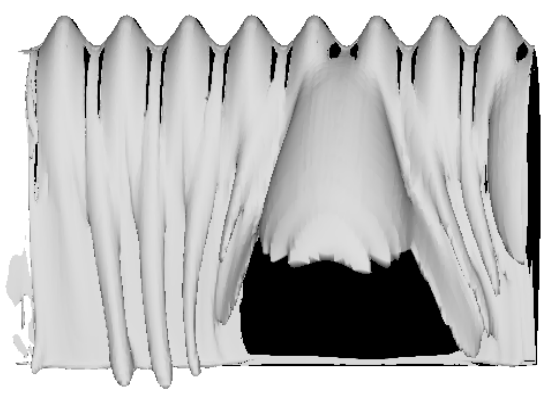

(e) 


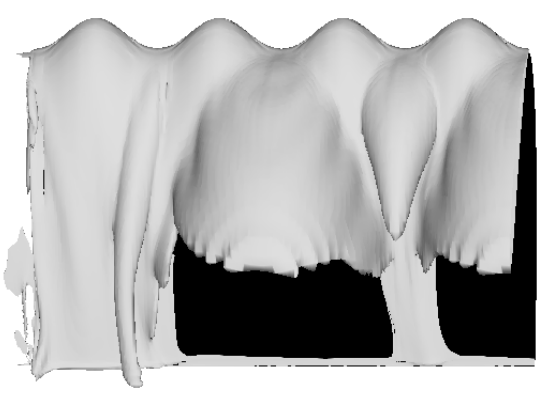

(c)

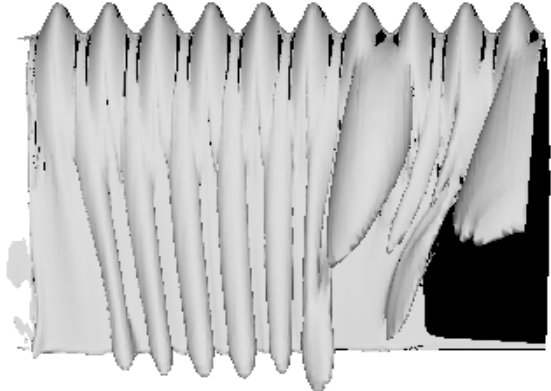

(f)

Fig. 9 Iso-surface of the spanwise vorticity on the upper surface at $\alpha=12^{\circ}$ for different wings; (a) smooth wing, (b) $\lambda=S / 2$, (c) $\lambda=S / 4$, (d) $\lambda=S / 6$, (e) $\lambda=S / 8$, and (f) $\lambda=S / 10$.

Fig. 10 and 11 show the limiting streamlines and the iso-surface of the spanwise vorticity on the upper surface of the wing, respectively, for the smooth wing and the wavy wings at $\alpha=20^{\circ}$. For the smooth wing, with increasing $\alpha$ from $12^{\circ}$ to $20^{\circ}$, the formation of the limiting streamlines dramatically changes, leading to the appearance of the helical nature of the limiting streamlines as shown in Fig. 13(a). Accordingly, the convergence lines move toward the leading edge, accompanied with return flow from the trailing edge. This pattern of limiting streamlines is consistent with the findings of Gregory et al. (1971) and Zanin et al. (2008). The components of a 3-dimensional vortical structure as shown in Fig. 11(a).

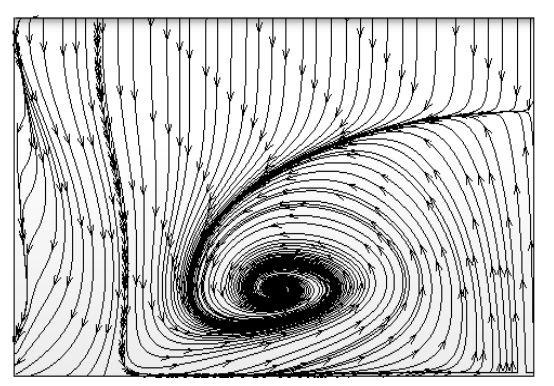

(a)

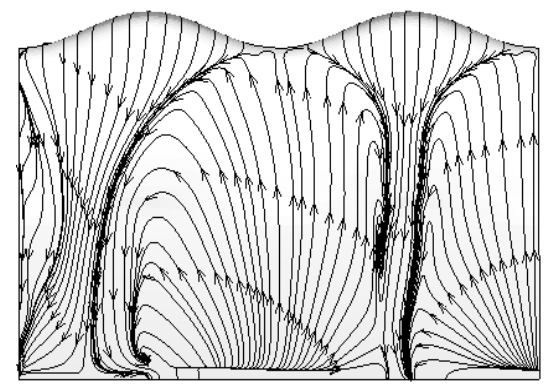

(b)

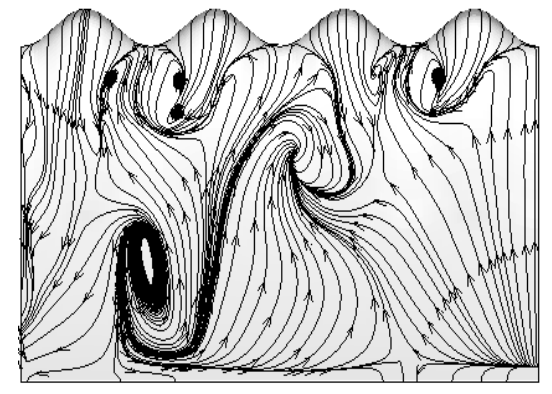

(c)

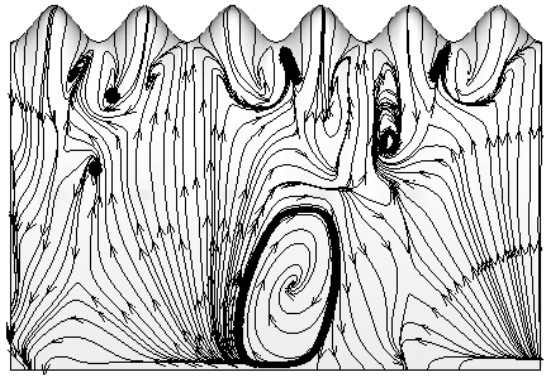

(d)

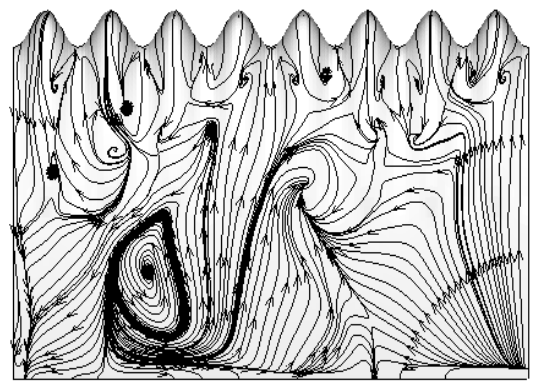

(e)

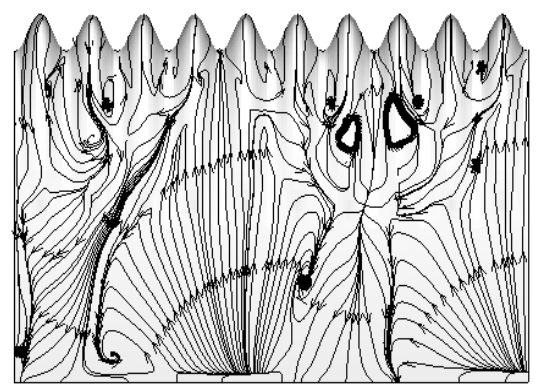

(f)

Fig. 10 Limiting streamlines on the upper surface at $\alpha=20^{\circ}$ for different wings; (a) smooth wing,

(b) $\lambda=S / 2$, (c) $\lambda=S / 4$, (d) $\lambda=S / 6$, (e) $\lambda=S / 8$, and (f) $\lambda=S / 10$. 


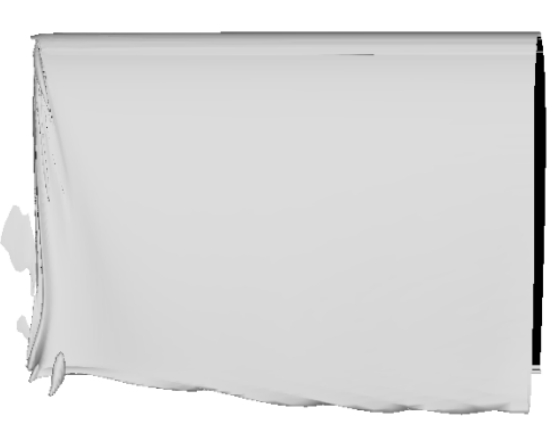

(a)

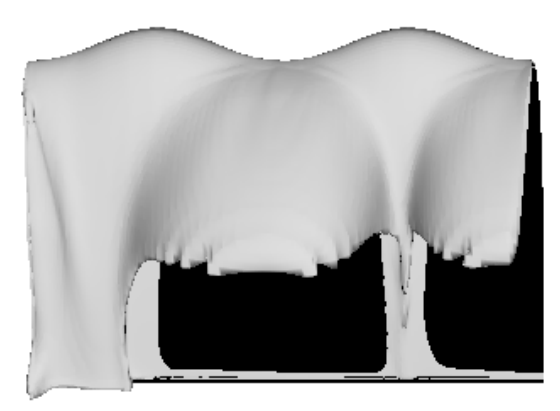

(b)

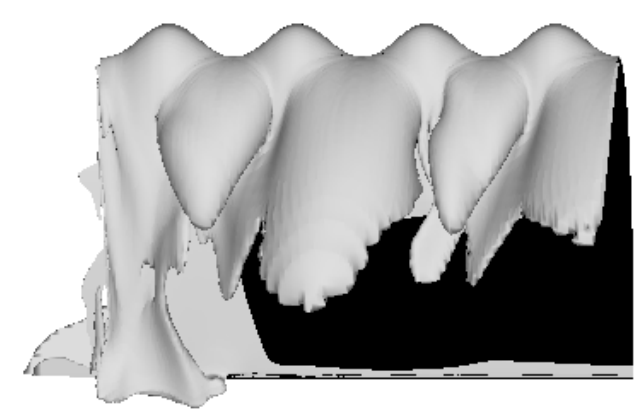

(c)

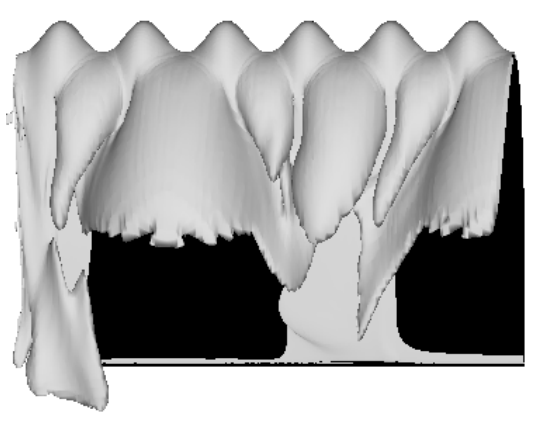

(d)

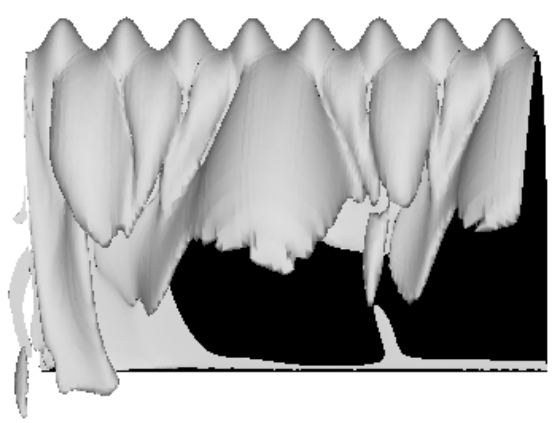

(e)

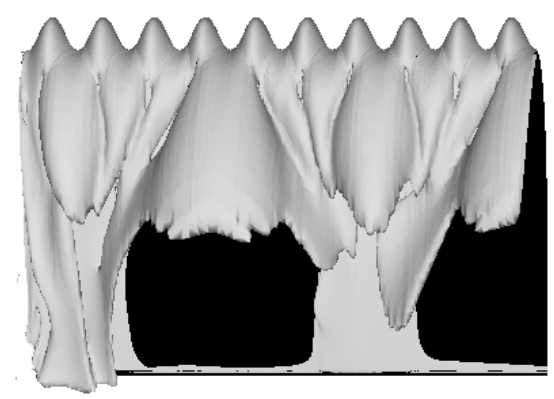

(f)

Fig. 11 Iso-surface of the spanwise vorticity on the upper surface at $\alpha=20^{\circ}$ for different wings; (a) smooth wing, (b) $\lambda=S / 2$, (c) $\lambda=S / 4$, (d) $\lambda=S / 6$, (e) $\lambda=S / 8$, and (f) $\lambda=S / 10$.

For wavy wings, as $\alpha$ increases from $12^{\circ}$ to $20^{\circ}$, the return flow covers wider region and the various convergence lines appear behind the troughs, forming much complicate limiting streamlines, which can be certified by comparing Figs. 8 (b) (f) and Figs. 10(b) (f) for $\alpha=12^{\circ}$ and $\alpha=20^{\circ}$, respectively. This augmentation of the return flow and within the diverged convergence lines of the limiting streamlines is identified by the large iso-surface of the spanwise vorticity as shown in Figs. 11(b) (f). Consequently, the similar formation among the wavy wings can support the achievement of similar values of the lift and drag coefficients as previously documented in Figs. 4(a) and 4(b).

Fig. 12 and 13 show the limiting streamlines and the iso-surface of the spanwise vorticity on the upper surface, respectively, for the smooth wing and wavy wings at $\alpha=24^{\circ}$. For the smooth wing, because the flow separates from the leading edge, the return flow covers most of the upper surface as shown in Fig. 12(a). This distribution of the limiting streamlines corresponds to the large iso-surface of spanwise vorticity that emerged from the leading edge as plotted in Fig. 13(a).

Generally, Miklosovic et al. (2004) and Fish and Lauder (2006) suggested that the waviness works as the vortex generator in the leading edge. Also, Yoon et al. (2011) presented the consistent results with the suggestion of Miklosovic et al. (2004) and Fish and Lauder (2006). The present results show that a the spiral formation of the limiting streamlines in the wavy troughs of the wavy wings modifies the separation line along the leading edge of the smooth wing, as shown in Figs. 12(b) (f). Successively, the large iso-surface of spanwise vorticity in the smooth wing is broken into an array of smaller iso-vorticity surfaces in the wavy leading edge region, which is much clearer with decreasing wavy length as observed in Figs. 13(b) (f). 


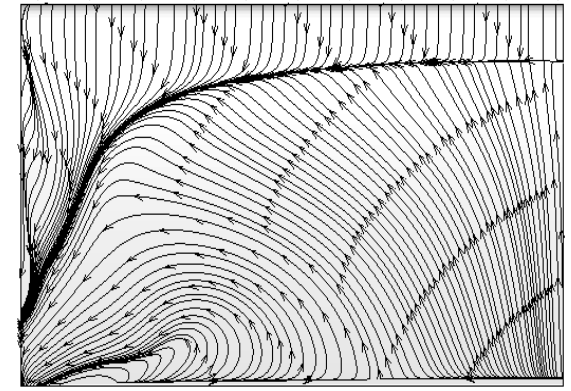

(a)

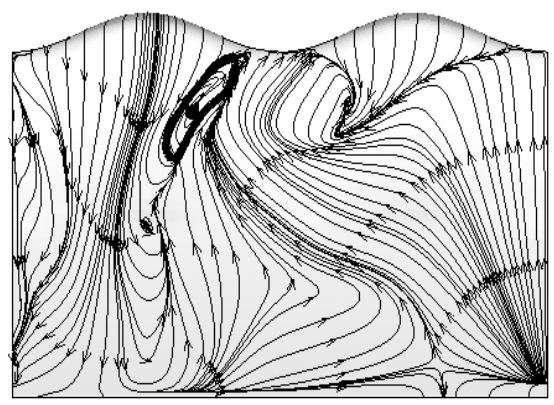

(b)

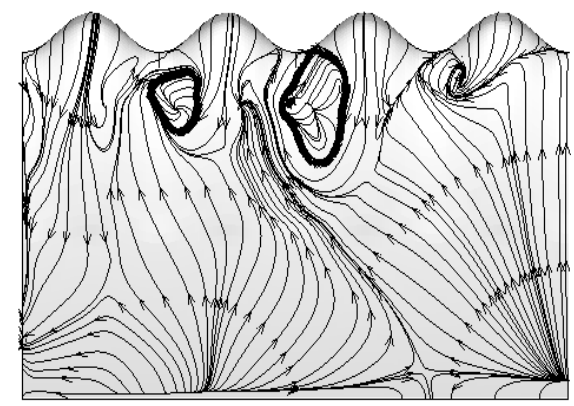

(c)

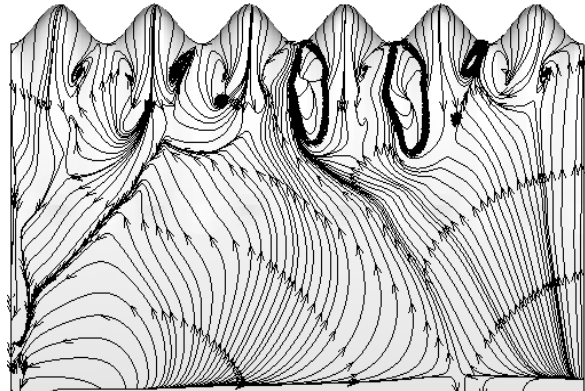

(d)

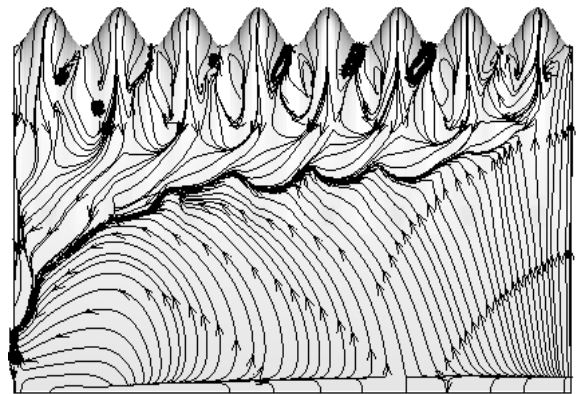

(e)

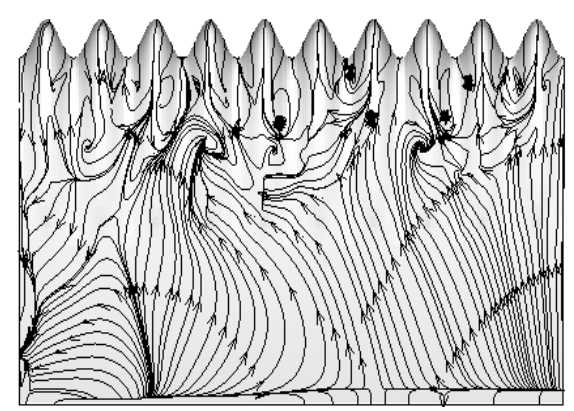

(f)

Fig. 12 Limiting streamlines on the upper surface at $\alpha=20^{\circ}$ for different wings; (a) smooth wing, (b) $\lambda=S / 2$, (c) $\lambda=S / 4$, (d) $\lambda=S / 6$, (e) $\lambda=S / 8$, and (f) $\lambda=S / 10$.

\section{CONCLUSIONS}

The present study numerically investigated the effect of the wavy leading edge on the hydrodynamic characteristics of three-dimensional rectangular wings with NACA 0020 section. Five different wave lengths of $S / 2, S / 4, S / 6, S / 8$ and $S / 10$ at fixed wavy amplitude of $0.05 C$ have been considered. Numerical simulations are performed at a wide range of the angle of attack $\left(0^{\circ} \leq \alpha \leq 40^{\circ}\right)$ at one Reynolds number of $10^{6}$.

In the range of $0^{\circ} \leq \alpha \leq 8^{\circ}$, the general distribution of $C_{P}$ for wavy wings is similar to that of the smooth wing and also the flow structures such as limiting streamlines and the spanwise vorticity are slightly altered behind the wavy region. As a result, the hydrodynamic forces were not much affected by the wavy leading edge regardless of the wave length.

However, when $8^{\circ}<\alpha<24^{\circ}$, the waviness considerably modified flow structures on the upper surface, forming the diverged convergence lines of the limiting streamlines identified by the large iso-surface of the spanwise vorticity. This pattern of the limiting streamline correlates with the relatively high $C_{P}$ distribution in the centre of the wing. Therefore, in this range of $8^{\circ}<\alpha<24^{\circ}$, irrespective of the wave length, the wavy wings provided the considerable loss of the lift, compared to the smooth wing. 
When $\alpha \geq 24^{\circ}$, the waviness contributed to forming the spiral formation of the limiting streamlines in the wavy troughs of the wavy wings and an array of smaller iso-vorticity surfaces in the wavy leading edge region. This modification of flow structures was limited very near the leading edge. Successively, $C_{P}$ distributions of wavy wings were generally similar to those of the smooth wing. Therefore, in this range of angles of attack, the lift coefficients of the smooth wing and the wavy wings are not much different.

\section{ACKNOWLEDGMENT}

This work was supported by the National Research Foundation of Korea (NRF) grant funded by the Korea government (MEST) through GCRC-SOP (No. 2011-0030662).

\section{REFERENCES}

Custodio, D., 2007. The effect of humpback whale-like leading edge protuberances on hydrofoil performance. MS. Worcester Polytechnic Institute, United States.

Fish, F.E. and Battle, J.M., 1995. Hydrodynamic design of the humpback whale flipper. Journal of Morphology, 225(1), pp.51-60.

Fish, F.E. and Lauder, G.V., 2006. Passive and active flow control by swimming fishes and mammals. Annual Review of Fluid Mechanics, 38, pp.193-224.

Fluent Inc, 2005. Fluent user's guide version 6.2.16. Lebanon, Fluent Inc.

Gregory, N., Quincey, V.G., O'Reilly, C.L. and Hall, D.J., 1971. Progress report on observations of three-dimensional flow patterns obtained during stall development on aerofoils, and on the problem of measuring two-dimensional characteristics. London, Ministry of Aviation Supply, Aeronautical Research Council, No. 1146.

Johari, H., Henoch, C., Custodio, D. and Levshin, A., 2007. Effects of leading-edge protuberances on airfoil performance. AIAA Journal, 45(11), pp. 2634-2642.

Molland, A.F. and Turnock, S.R., 1993. Wind tunnel tests on the influence of propeller loading on ship rudder performance: Four quadrant operation, low and zero speed operation. Ship Science Report No. 64, University of Southampton.

Miklosovic, D.S., Murray, M.M., Howle, L.E. and Fish, F.E., 2004. Leading-edge tubercles delay stall on humpback whale (Megaptera novaeangliae) flippers. Physics of fluids, 16(5), pp. L39-L42.

Miklosovic, D.S., Murray, M.M. and Howle, L.E., 2007. Experimental evaluation of sinusoidal leading edges. Journal of Aircraft, 44(4), pp.1404-1407.

Murray, M.M., Miklosovic, D.S., Fish, F.E. and Howle, L.E., 2005. Effects of leading edge tubercles on a representative whale flipper model at various sweep angles. In: Conference Proceedings of the 14th International Symposium on Unmanned Untethered Submersible Technology. August, Durham New Hampshire, AUSI.

van Nierop, E.A., Alben, S. and Brenner, M.P., 2008. How bumps on whale flippers delay stall: An aerodynamic model. Physical Review Letters, 100(5), 054502.

Watts, P. and Fish, F.E., 2001. The influence of passive, leading edge tubercles on wing performance. Proceedings of the Twelfth International Symposium on Unmanned Untethered Submersible Technology. Autonomous Undersea Systems Institute, Durham, New Hampshire.

Yoon, H.S., Hung, P.A., Jung, J.H. and Kim, M.C., 2011. Effect of the wavy leading edge on hydrodynamic characteristics for flow around low aspect ratio wing. Computers \& Fluids, 49(1), pp.276-289.

Zanin, B.Y., Zverkov, I.D., Kozlov, V.V. and Pavlenko, A.M., 2008. Vortex structure of separated flows on model wings at low freestream velocities. Fluid Dynamics, 43(6), pp.938-944. 